\title{
РОЛЬ ПОЛИМОРФИЗМА ГЕНА ВRСА1 У ПАЦИЕНТОВ С РЕЦИДИВИРУЮЩИМ РАКОМ ЯИЧНИКОВ
}

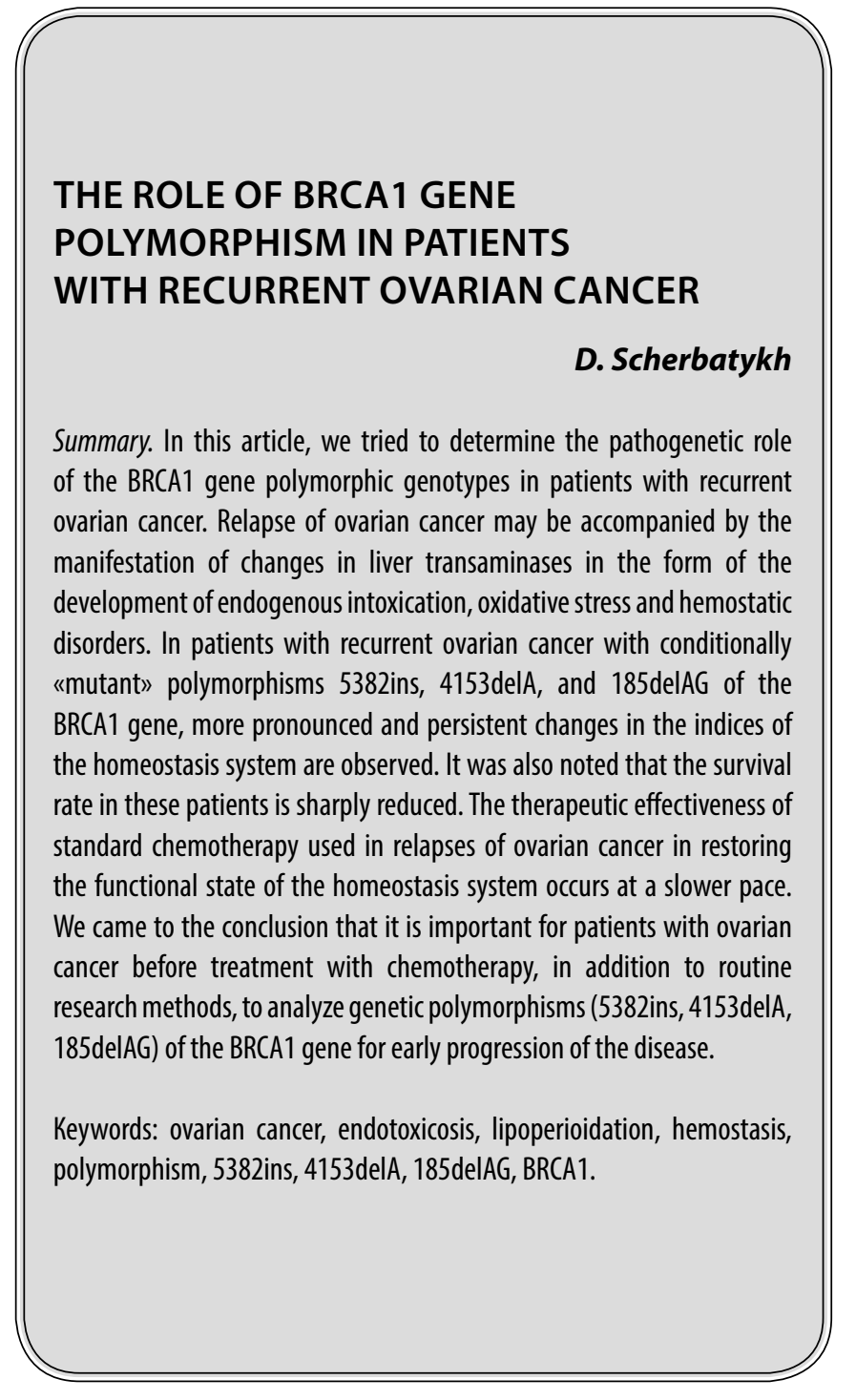

\section{Введение}

B последние десятилетия рецидивирующий рак яичников (РЯ) является актуальным вопросом в медицинской практике не только в России, но и во всем мире $[1,2]$.

По эпидемиологическим исследованиям отмечено, что частота заболеваемости рака яичников в Российской Федерации составляет 26,4\%. При этом рак яичников занимает 9-е место среди злокачественных патологий женского населения (около 4,5\%), 3-е место в структуре опухолей заболеваний половых органов (около 24,6\%)

\author{
Щербатых Дарья Евгеньевна \\ Аспирант, Санкт-Петербургский \\ научно-клинический чентр специализированных видов \\ медицинской помощи (онкология); \\ ФГБОУ ВО «Санкт-Петербургский государственный \\ педиатрический медичинский университет» \\ Минздрава России \\ scherbatykh.d@bk.ru
}

Аннотация. В этой статье мы попытались определить патогенетическую роль полиморфных генотипов гена BRCA1 у пациентов с рецидивирующим раком яичников. Рецидив рака яичников может сопровождаться проявлением изменений трансаминаз печени в виде развития эндогенной интоксикации, окислительного стресса и гемостатическими нарушениями. У пациентов с рецидивами рака яичников, имеющих условно «мутантные» полиморфизмы 5382ins, 4153delA и 185delAG гена BRCA1 наблюдаются 60лее выраженные и стойкие изменения показателей системы гомеостаза. Также отмечено, что показатель выживаемости у этих пациентов резко снижается. Терапевтическая эффективность стандартной химиотерапии, применяемой при рецидивах рака яичников, в восстановлении функционального состояния системы гомеостаза происходит замедленным темпом. Раннее включение гормонотерапии у пациентов с рецидивирующим раком яичников приводит к улучшению общей эффективности от стандартной химиотерапии: сравнительно быстро уменьшается выраженность эндогенной интоксикации и активности перекисного окисления липидов и оперативно восстанавливается состояние свертывающей системы, и как итог - снижению показателя летальности и улучшению выживаемости. Это особенно наблюдается у пациентов с генетическими полиморфизмами исследованного гена. Пришли к выводам, что пациентам с раком яичников до лечения химиопрепаратами помимо дополнительно рутинных методов исследования важно проводить анализ генетических полиморфизмов (5382ins, 4153delA, $185 \mathrm{deIAG)}$ гена BRCA1 для раннего прогрессирования течения заболевания.

Ключевые слова: рак яичников, эндотоксикоз, липопериокисление, гемостаз, полиморфизм, 5382ins, 4153delA, 185delAG, BRCA1.

и 1-е место по летальному исходу от него (около 33,9\%) $[1,2]$.

Следует отметить, частота рецидивирующей формы заболевания у пациентов раком яичников остается достаточно высокой, составляя 70,0-77,9\%. Средняя продолжительность жизни пациентов с рецидивирующим РЯ при анеуплоидном виде опухоли составляет 11,9 мес., а при диплоидном - 20,8 мес [2].

Важная роль в утяжелении течения болезни и развитии жизненно-серьезных осложнений отводится печеночной депрессии. Одними из проявлений расстройства 
этого органа является формирования эндогенной интоксикации, активация свободно радикального окисления и изменения системы гемостаза. Комплексные нарушения, обусловленные эндотоксикозом, оксидативным стрессом и активацией свертывания, могут явиться основными причинами серьезных морфофункциональных нарушений различных органов и систем, летальных исходов $[3,4]$.

В последнее время появляется наглядная необходимость в исследовании молекулярной роли ряда генов в развитии и рецидива рака яичников [3].

Ген BRCA1 контролирует белок - супрессор опухолевого процесса, который играет важную роль в восстановлении поврежденной ДНК за счёт гомологичной рекомбинации и сохранении стабильности генетического аппарата клетки. Существует разновидность мутаций гена $B R C A 1$, имеющих связь не только с опухолями яичников, но и других органов (молочной железы, поджелудочной железы, предстательной железы и др. [4].

Цель исследования. Определить патогенетическую роль полиморфных генотипов гена $B R C A 1$ у пациентов с рецидивирующим раком яичников.

\section{Материалы и методы исслеАования}

Проводилось обследование 50 пациентов с рецидивирующим раком яичников. Они разделены на две группы. Первая группа (сравнения, $\mathrm{n}=25$ ) - больные с рецидивирующим раком яичников на фоне стандартной схемы лечения (химиотерапии), их возраст был $45,5 \pm 6,28$, вторая (основная, $\mathrm{n}=30$ ) - аналогична первой, но на фоне комплексной схемы лечения (химиотерапии + гормонотерапии «Тамоксифен», возраст - 47,1 $\pm 5,97$.

Критерии применения пациентов в данную работу: письменное согласие на участие в это исследование, патоморфологическая верификация диагноза «Рак яичника. Рецидив», наличие маркера рака яичников - CA125, возрастая категория от 22 до 69 лет, сопутствующие патологии легкой степени.

Критерии исключения из работы: персональный отказ от участия, нарушение больным протокола исследования, возраст старше 69 и моложе 22 лет, отсутствие маркера рака яичников - СА125, использование хирургической терапии, вредные привычки, тяжелые сопутствующие заболевания (инфекционных, соматических, психологических), беременность.

Параллельно обследованы пациенты (n=35) для сравнения изученных показателей с референтными значениями в возрасте от 23 до 48 лет.
Пациентам первой и второй групп назначена химио-

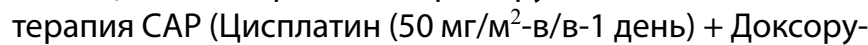

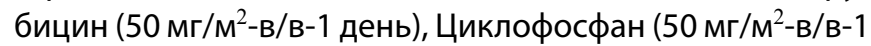
день), (50 мг/м²-в/в-1 день)) по 4-м циклам каждые 3 недели. Однако в схему лечения основной группы дополнительно включена гормонотерапия (Тамоксифен, 20 мг, внутрь, 1 раз в день).

\section{В исследовании приняты следующие методы.}

Классификация стадий заболевания исследуемых пациентов с помощью трехступенчатой системе и FIGO [5].

Определены интенсивность синдрома эндотоксикоза по уровню показателей гидрофобных метаболитов (эффективной концентрации альбумина (ЭКА, г/л), резерва связывания альбумина (РСА, усл.ед.)) в плазме крови; активность перекисного окисления липидов (ПОЛ) по значению диеновых конъюгатов (ДК, у.е./мг липидов) и малонового диальдегида (МДА, нМоль/г белка); функциональная активность системы коагуляции крови по параметрам тромбоэластографа TEG ${ }^{\circledR} 5000$ (США) реактивного времени ( $\mathrm{R}$, мин) и коагуляционного индекса $(\mathrm{Cl}, \%)$ при помощи тромбоэластографа TEG ${ }^{\circledR} 5000$ (США) [6]. Данные исследования проведены в промежутке от 2 до 4 часов после назначенного лечения.

Молекулярное анализирование полиморфизма гена BRCA1 5382ins, 4153delA и 185delAG проведено при использовании амплификатора CFX96 Touch $^{\text {TM }}$ в реальном времени (США) [7].

Срок исследовательского наблюдения - 3 года после лечения первичного рака яичника.

Результаты, полученные по ходу исследования, статистически обработаны программами Microsoft Word, Microsoft Excel XP (2013) и «Statistica 13.3 Trial» с использованием критерия $X^{2}$ Пирсона, Стьюдента и Фишера, и величины отношения шансов (OR, 95\%).

\section{Результаты исслеАования и их обсужАение}

Пациентам исследования как первой, так и второй группы выполнялись циторедуктивные операции при выявлении первичного рака яичника.

При изучении срока диагностики рецидива РЯ отмечено, что срок вуалировался от 9 мес. до 30 мес., а в среднем - 16,2 $\pm 2,69$ мес.

При изучении генетических исследований установлено, что частота встречаемости полиморфизма $5382 \mathrm{ins}, 4153 \mathrm{del} A$ и $185 \mathrm{del} A G$ гена $B R C A 1$ в первой 
Таблица 1. Распределение исследуемых пациентов по виду мутации гена $B R C A 1$

\begin{tabular}{|l|l|l|l|}
\multirow{2}{*}{ Вид мутации } & Группы исследования $\mathbf{n}(\%)$ & \multicolumn{2}{|l|}{} \\
\cline { 2 - 4 } & Нормы (n=35) & первая $(\mathbf{n}=\mathbf{2 5})$ & Вторая $(\mathbf{n}=\mathbf{2 5})$ \\
\hline 5382ins & Не обнаружен & $5(20,0)$ & $5(24,0)$ \\
\hline 185delAG & Не обнаружен & $4(16,0)$ & $2(8,0)$ \\
\hline
\end{tabular}

Таблица 2. Распределение исследуемых пациентов по FIGO и трехступенчатой системе (Grade) в ассоциации с полиморфизмами гена $B R C A 1$

\begin{tabular}{|c|c|c|c|c|c|c|}
\hline \multirow{3}{*}{ Тип мутации } & \multicolumn{6}{|c|}{ Вид опухолевого процесса n (\%) } \\
\hline & \multicolumn{3}{|l|}{ FIGO } & \multicolumn{3}{|l|}{ Grade } \\
\hline & 1 & II & III & G1 & G2 & G3 \\
\hline \multicolumn{7}{|l|}{ Первая ( $n=25)$} \\
\hline 5382ins & 0 & $4(16,0)$ & $1(4,0)$ & $2(8,0)$ & $3(12,0)$ & 0 \\
\hline 4153delA & 0 & $3(12,0)$ & $1(4,0)$ & $1(4,0)$ & $2(8,0)$ & $1(4,0)$ \\
\hline 185delAG & 0 & $1(4,0)$ & $2(8,0)$ & $2(8,0)$ & $1(4,0)$ & 0 \\
\hline Не обнаружено & $8(32,0)$ & $5(20,0)$ & 0 & $2(8,0)$ & $4(16,0)$ & $7(28,0)$ \\
\hline \multicolumn{7}{|l|}{ Вторая $(n=25)$} \\
\hline 5382ins & 0 & $3(12,0)$ & $3(12,0)$ & $3(12,0)$ & $2(8,0)$ & $1(4,0)$ \\
\hline 4153delA & 0 & $4(16,0)$ & $1(4,0)$ & $2(8,0)$ & $3(12,0)$ & 0 \\
\hline 185delAG & 0 & $1(4,0)$ & $1(4,0)$ & $1(4,0)$ & $1(4,0)$ & 0 \\
\hline Не обнаружено & $9(36,0)$ & $3(12,0)$ & 0 & 0 & $2(8,0)$ & $10(40,0)$ \\
\hline
\end{tabular}

группе составила $32,0,36,0$ и $28,0 \%$, а во второй - 40,0, 44,0 и $20,0 \%$ (табл. 1).

У части исследуемых больных не обнаружены мутации, в группе сравнения - $13(52,0 \%)$ и основной -12 $(48,0 \%)$ (табл. 1).

Далее, группы исследования были разделены на подгруппы в зависимости от наличия и отсутствия мутаций: первая группа - на подгруппу 1 ( $\mathrm{n=12}$, пациенты c 5382ins, 4153delA и $185 \mathrm{del} A G$ гена $B R C A 1)$ и подгруппу 2 ( $\mathrm{n}=13$, больные без мутации гена $B R C A 1)$; вторая - на подгруппу $3(\mathrm{n}=13$, пациенты с 5382ins, 4153 del $A$ и 185 del $A G$ гена $B R C A 1)$ и подгруппу 4 ( $n=14$, больные без мутации гена $B R C A 1)$.

Гистологические исследования показали, что у пациентов с рецидивом РЯ показаны разные виды опухолевого процесса по FIGO и трехступенчатой системе, которые были связаны с полиморфизмами гена $B R C A 1$.

у пациентов подгруппы 1 (с мутациями 5382ins, 4153 delA и 185 delAG гена BRCA1) определены II и III стадии по системе FIGO у 16,0 и 4,0, 12,0 и 4,0 и 4,0 и 8,0\% соответственно. У больных подгруппы 2 (без полимор- физма гена $B R C A 1)$ регистрировались 1-я и 2-я стадии у 32,0 и $20,0 \%$ соответственно (табл. 2).

У пациентов основной группы установлены вторая и третья стадии FIGO системы у пациентов подгруппы 3 по 12,0 и 12,0, 16,0 и 4,0 и 4,0 и 4,0\% соответственно. Больные подгруппы 4 имели I и ІІ стадии по 36,0 и 12,0\% (табл. 2).

При распределении пациентов первой группы по трехступенчатой системе установлено, что высокодифференцированный рак обнаружен у больных с генетическими изменениями (5382ins, $1153 \mathrm{del}$ и и $185 \mathrm{del} A G)$ гена $B R C A 1$ в 8,0, 4,0 и 8,0\%, а также у 8,0\% пациентов без мутации. Умереннодифференцированная форма РЯ наблюдена и у пациентов подгруппы $1-$ у 12,0, 8,0, 4,0\%, и подгруппы 2 - у 16,0\%. Низкодифференцированный рак отмечен у 28,0\% пациентов подгруппы 2 и 4,0\% c 4153delA полиморфизмом (табл. 2).

При анализе распределения дифференцировки РЯ у больных второй группы по трехступенчатой системе показано, что G1 регистрирована лишь в подгруппы 3 у 12,0, 8,0 и 4,0\% соответственно (табл. 2). G2 вид дифференцировки опухоли отмечен у пациентов подгруппы 3 
Таблица 3. Параметры системы гомеостаза при рецидиве РЯ на фоне лечения

\begin{tabular}{|c|c|c|c|c|c|c|}
\hline \multirow{2}{*}{ Показатель } & \multirow{2}{*}{$\begin{array}{l}\text { Норма } \\
(\mathrm{n}=15)\end{array}$} & \multirow{2}{*}{$\begin{array}{l}\text { Группы } \\
\text { исследования }\end{array}$} & \multicolumn{4}{|c|}{ Цикл лечения } \\
\hline & & & 1-й & 2-й & 3-й & 4-й \\
\hline \multirow{2}{*}{ OKA } & \multirow{2}{*}{$\begin{array}{l}44,7 \\
\pm 5,11 \\
\end{array}$} & I $(n=25)$ & $35,4 \pm 2,83$ & $34,6 \pm 2,72$ & $34,9 \pm 2,89$ & $36,7 \pm 3,15$ \\
\hline & & II $(n=25)$ & $40,1 \pm 4,42$ & $39,8 \pm 4,88$ & $39,4 \pm 4,26$ & $40,5 \pm 4,13$ \\
\hline \multirow{2}{*}{ ЭКА } & \multirow{2}{*}{$\begin{array}{l}38,5 \\
\pm 4,42 \\
\end{array}$} & I $(n=25)$ & $29,4 \pm 3,44$ & $30,5 \pm 4,91$ & $28,5 \pm 3,54$ & $29,9 \pm 3,37$ \\
\hline & & II $(n=25)$ & $33,4 \pm 4,12$ & $34,1 \pm 3,65$ & $33,8 \pm 3,12$ & $34,5 \pm 3,2$ \\
\hline \multirow{2}{*}{ ДК } & \multirow{2}{*}{$\begin{array}{l}207,2 \\
\pm 10,36 \\
\end{array}$} & I $(n=25)$ & $275,1 \pm 11,44$ & $268,2 \pm 8,56$ & $271,1 \pm 12,52$ & $269,4 \pm 12,11$ \\
\hline & & II $(n=25)$ & $235,4 \pm 12,5$ & $229,2 \pm 10,6$ & $231,1 \pm 11,8$ & $234,0 \pm 10,5$ \\
\hline \multirow{2}{*}{ МДА } & \multirow{2}{*}{$\begin{array}{l}2,31 \\
\pm 0,07\end{array}$} & I $(n=25)$ & $2,89 \pm 0,15$ & $2,91 \pm 0,05$ & $2,84 \pm 0,09$ & $2,93 \pm 0,11$ \\
\hline & & II $(n=25)$ & $2,56 \pm 0,12$ & $2,49 \pm 0,14$ & $2,48 \pm 0,16$ & $2,53 \pm 0,09$ \\
\hline \multirow{2}{*}{$\mathrm{R}$} & \multirow{2}{*}{$\begin{array}{l}5,72 \\
\pm 0,24\end{array}$} & I $(n=25)$ & $4,25 \pm 0,12$ & $4,30 \pm 0,56$ & $4,12 \pm 0,13$ & $4,32 \pm 0,18$ \\
\hline & & II $(n=25)$ & $4,99 \pm 0,14$ & $5,12 \pm 0,17$ & $4,92 \pm 0,15$ & $4,95 \pm 0,16$ \\
\hline \multirow{2}{*}{$\mathrm{Cl}$} & \multirow{2}{*}{$\begin{array}{l}1,18 \\
\pm 0,09\end{array}$} & I $(n=25)$ & $1,34 \pm 0,11$ & $1,35 \pm 0,04$ & $1,33 \pm 0,12$ & $1,35 \pm 0,15$ \\
\hline & & II $(n=25)$ & $1,22 \pm 0,13$ & $1,18 \pm 0,11$ & $1,20 \pm 0,10$ & $1,19 \pm 0,11$ \\
\hline
\end{tabular}

у 8,0, 12,0 и 4,0\% и подгруппы 4 - у 8,0\%. G2 форма (низкодифференцированная) обнаружена у 40,0 подгруппы 4 и 4,0\% у пациентов подгруппы 3 (табл. 2).

Таким образом, наличие генетических мутаций 5382ins, 4153delA u 185delAG гена BRCA1 сопровождается агрессивными формами РЯ (табл. 2).

Во время проведения терапевтических циклов химиотерапевтического лечения были оценены некоторые показатели системы гомеостаза (эндотоксикоза, ПОЛ и системы гемостаза).

По результатам работы установлено, что ранний период введения пациентов с рецидивирующим раком яичников характеризуется развитием эндогенной интоксикации. Отмечено снижение содержания гидрофобных продуктов (ОКА и ЭКА) после всех курсов лечения как в первой группе (на 22,1-21,9\% $(p=0,01))$, так и второй (на 10,2-11,8\% ( $p=0,01)$ (табл. 3).

У пациентов первой группы после введения стандартной химиотерапии содержание ДК и МДА превышало группу нормы на 1-2-3-4-м этапах лечения на 32,730,9 и $25,6-23,4 \%(p=0,01)$.

Во второй группе, пациентам которой была назначено комплексное лечение, выраженность процессов липопереокисления была меньше: уровень диеновых конъюгатов и малонового диальдегида был повышен на $10,5-11,5$ и $11,3-9,5 \%(p=0,01)$

Регистрировано, что рецидив РЯ сопровождается значительными расстройствами системы гемостаза в виде гиперкоагуляции и гипофибринолиза (табл. 3) $[9,10]$.

Так, у пациентов группы сравнения отмечено сокращение реактивного времени, показывающего активность свертывающей способности крови, на 25,4-27,7\% ( $p=0,01)$ и удлинение $\mathrm{Cl}$ (показатель фибринолиза) на $21,8-22,7 \%(p=0,01)$.

У пациентов основной группы значение R было снижено на всех циклах лечения на 12,4-13,6\% ( $p=0,01)$, а коагуляционного индекса - удлинение - на 10,9-9,1\% $(p=0,01)$.

Необходимо обратить внимание на то, что формирование расстройства системы гомеостаза у исследуемых пациентов с рецидивирующим РЯ статистически сопряжено с видом назначенной терапии. Результаты сравнительного анализа подтверждают, что интенсивность отклонений исследуемых показателей регистрирована более выражена у больных первой группы (с стандартным методом лечения) по сравнению с основной группой (на фоне комплексной терапии): значение ЭКА и РСА - было ниже на 33,1-15,0 и 18,5-16,1\% ( $p=0,01)$, ДК и МДА - выше - на 14,5-13,0 и 12,8-13,5\% ( $p=0,01)$, $\mathrm{R}$ - выше - на 17,4-19,2\% ( $\mathrm{p}=0,01), \mathrm{Cl}$ - ниже - на 12,5$11,9 \%(p=0,01)$.

При анализировании связи изучаемых полиморфизмов гена BRCA1 исследуемых параметров в условиях рецидивов рака яичников на фоне стандартной химиотерапии установлено, что в подгруппе 2, наблюдаются наименьшие расстройства в системе гомеостаза на протяжении всех лечебных циклов. У пациентов данной 
Таблица 4. распределение исследуемых пациентов выживаемости в ассоциации с полиморфизмами гена BRCA1

\begin{tabular}{|c|c|c|c|c|c|c|c|c|}
\hline \multirow{3}{*}{$\begin{array}{l}\text { Показатель } \\
\text { выживаемо- } \\
\text { сти, год }\end{array}$} & \multicolumn{8}{|c|}{ Группы исследования n (\%) } \\
\hline & \multicolumn{4}{|c|}{ Первая ( $\mathrm{n}=\mathbf{2 5})$} & \multicolumn{4}{|c|}{ Вторая ( $\mathrm{n}=25)$} \\
\hline & 5382ins & 4153 delA & 185delAG & Нет & 5382ins & 4153delA & 185delAG & Нет \\
\hline Первый & $2(8,0)$ & $1(4,0)$ & $1(4,0)$ & $1(4,0)$ & $1(4,0)$ & 0 & 0 & 0 \\
\hline Второй & $3(12,0)$ & $2(8,0)$ & $2(8,0)$ & $2(8,0)$ & $2(8,0)$ & $2(8,0)$ & 0 & $1(4,0)$ \\
\hline Трети & 0 & $1(4,0)$ & 0 & $6(24,0)$ & $1(4,0)$ & $1(4,0)$ & $1(4,0)$ & $3(12,0)$ \\
\hline
\end{tabular}

подгруппы (по сравнению с подгруппы 1) концентрации ОКА и ЭКА были выше на 13,1-14,7 и 12,4-15,1\% $(p<0,05)$, ДК и МДА - ниже на 14,1-13,2\% (p<0,05), R выше - на 13,5-15,7\% ( $p=0,01), \mathrm{Cl}$ - ниже — на 13,1$14,8 \%(p=0,01)$.

При изучении сопряженности генетических мутаций (5382ins, 4153delA и 185delAG) гена BRCA1 с терапевтической эффективностью комбинированного метода лечения было установлено, что применение гормонотерапии дополнительно в стандартную схему химиотерапии повышает общую лечебную эффективность рецидива РЯ и оказывает существенное воздействие у больных с патологическими полиморфизмами гена BRCA1 [9]. Сравнительный анализ в соответствующих подгруппах показал, что включение Тамоксифена в базисную терапию ведет к снижению интенсивности (по уровню ОКА и ЭКА) на 14,1-16,4 и 13,7-15,3\% $(p<0,05)$ и активности ПОЛ (ДК и МДА на 14,8-16,9 и 15,2-17,2\% $(p<0,05))$ и восстановлению нарушения системы гемостаза (реактивное время увеличивалось на 14,1-15,3\% ( $<<0,05)$, показатель фибринолиза сократился на $13,7-16,1 \%(p<0,05))$.

При изучении выживаемости больных исследования установлено, что летальный исход наблюдается больше у пациентов с мутациями гена $B R C A 1$. Однако включение гормонотерапии сравнительно улучшает выживаемость (на 21,6\%, p=0,01) (табл. 4)

\section{ВывОАы}

1. Рецидивы рака яичников сопровождается печеночной депрессии, которая проявляется в виде развития эндогенной интоксикации, окислительного стресса и гемостатическими нарушениями.

2. У пациентов с рецидивами рака яичников, имеющих условно «мутантные» полиморфизмы 5382ins, 4153delA и 185delAG гена BRCA1 наблюдаются более выраженные и стойкие изменения показателей системы гомеостаза. Также отмечено, что показатель выживаемости у этих пациентов резко снижается.

3. Терапевтическая эффективность стандартной химиотерапии, применяемой при рецидиве рака яичника, в восстановлении функционального состояния системы гомеостаза происходит замедленным темпом.

4. Раннее включение гормонотерапии у пациентов с рецидивирующим раком яичников приводит к улучшению общей эффективности стандартной химиотерапии: сравнительно быстро уменьшается выраженность эндогенной интоксикации и активности перекисного окисления липидов и оперативно восстанавливаются состояние свертывающей системы, и как итог - снижению показателя летальности и улучшению выживаемости. Это особенно наблюдается у пациентов с генетическими полиморфизмами исследованного гена.

\section{ЛИТЕРАТУРА}

1. Владимирова Л.Ю., Сторожакова А.Э., Калабанова Е.А., Вереникина Е.В., Кабанов С.Н., Светицкая Я.В., Саманева Н.Ю., Тихановская Н.М., Новоселова К.А., Селезнева 0.Г., Тишина А.В. Опыт применения бевацизумаба в поддерживающей терапии у больных раком яичников // Южно-российский онкологический журнал. 2020: 3. URL: https://cyberleninka.ru/article/n/opyt-primeneniya-bevatsizumaba-v-podderzhivayuschey-terapii-u-bolnyh-rakom-yaichnikov (дата обращения: 12.04 .2021$)$.

2. Torre L.A., Trabert B., DeSantis C.E., Miller K.D., Samimi G., Runowicz C.D., Gaudet M.M., Jemal A., Siegel R.L. Ovarian cancer statistics, 2018 // CA Cancer J Clin. 2018; 68: 4: 284-296. Doi: 10.3322/caac.21456.

3. Здрок В.С., Жигимонт А.В. Выявление закономерностей в изменении клинико-лабораторных показателей при раке предстательной железы различной гистоморфологической градации // FORCIPE. 2020: S. URL: https://cyberleninka.ru/article/n/vyyavlenie-zakonomernostey-v-izmenenii-kliniko-laboratornyhpokazateley-pri-rake-predstatelnoy-zhelezy-razlichnoy (дата обращения: 12.04.2021).

4. Румянцев А.А., Тюляндина А.С. Практические аспекты лечения рецидивов brca-ассоциированного рака яичников // MC. 2019; 19. URL: https://cyberleninka. ru/article/n/prakticheskie-aspekty-lecheniya-retsidivov-brca-assotsiirovannogo-raka-yaichnikov (дата обращения: 12.04.2021). 
5. Набиева Ф.С., Мухамеджанова М.X. Особенности диагностики и лечение раннего рака яичников у молодых женщин // Вестник CMУC74. 2016; 3: 14. URL: https://cyberleninka.ru/article/n/osobennosti-diagnostiki-i-lechenie-rannego-raka-yaichnikov-u-molodyh-zhenschin (дата 0бращения: 12.04.2021).

6. Lheureux S., Braunstein M., Oza A.M. Epithelial ovarian cancer: Evolution of management in the era of precision medicine. CA Cancer J Clin. 2019; 69: 4: 280-304. Doi: 10.3322/caac.21559.

7. Liu H., Yang L. Dynamic change of depression and anxiety after chemotherapy among patients with ovarian cancer. Medicine (Baltimore). 2019; 98: 31: e16620. Doi: 10.1097/MD.0000000000016620.

8. Карелина 0.Б., Артымук Н.В., Фетисова Т.И. Факторы риска рака яичника и возможные превентивные стратегии. Фундаментальная и клиническая медицина. 2018; 3:3:91-96.

9. Marí-Alexandre J., Carcelén A.P, Agababyan C., Moreno-Manuel A., García-Oms J., Calabuig-Fariñas S., Gilabert-Estellés J. Interplay Between MicroRNAs and Oxidative Stress in Ovarian Conditions with a Focus on Ovarian Cancer and Endometriosis. Int J Mol Sci. 2019; 20: 21: 5322. Doi: 10.3390/ijms20215322.

10. Антонеева И.И., Генинг Т.П., Арсланова Д.Р., Сидоренко Е.Г. Сравнительная характеристика оксидативного стресса при раке шейки матки на разных клинических стадиях заболевания. Опухоли женской репродуктивной системы. 2011; 4: 55-58. Doi.org/10.17650/1994-4098-2011-0-4-55-58.

11. Павлова B.Ю., Ливадный Е.С. Биотехнология car-t и новые возможности лечения опухолевых заболеваний // Клиническая онкогематология. 2021; 1. URL: https://cyberleninka.ru/article/n/biotehnologiya-car-t-i-novye-vozmozhnosti-lecheniya-opuholevyh-zabolevaniy (дата обращения: 12.04.2021).

12. Penson R.T., Valencia R.V., Cibula D., Colombo N., Leath C.A. 3rd, Bidziński M, Kim J.W., Nam J.H., Madry R., Hernández C., Mora P.A.R., Ryu S.Y., Milenkova T., Lowe E.S., Barker L., Scambia G. Olaparib Versus Nonplatinum Chemotherapy in Patients With Platinum-Sensitive Relapsed Ovarian Cancer and a Germline BRCA1/2 Mutation (SOLO3): A Randomized Phase III Trial. J Clin Oncol. 2020; 38: 11: 1164-1174. Doi: 10.1200/JC0.19.02745.

13. Kim S.I., Lee M., Kim H.S., Chung H.H., Kim J.W., Park N.H., Song Y.S. Effect of BRCA mutational status on survival outcome in advanced-stage high-grade serous ovarian cancer // J Ovarian Res. 2019; 12: 1: 40. Doi: 10.1186/s13048-019-0511-7.

(c) Щербатых Дарья Евгеньевна ( scherbatykh.d@bk.ru ).

Журнал «Современная наука: актуальные проблемы теории и практики»

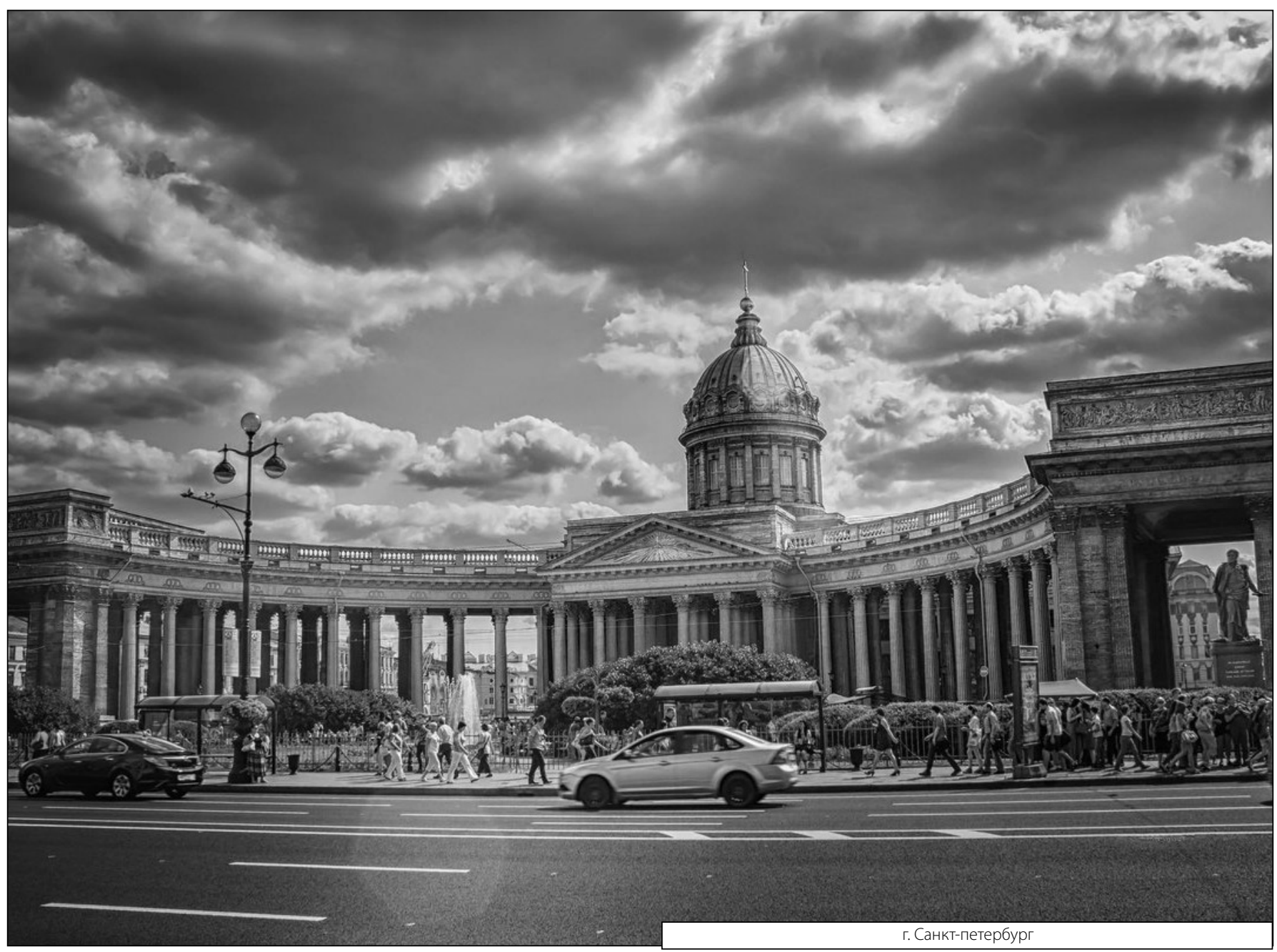

